\title{
Corela
}

Cognition, représentation, langage

HS-6 | 2007

Cognition, discours, contextes

\section{La linguistique cognitive et Humboldt}

\section{Anne-Marie Chabrolle-Cerretini}

\section{OpenEdition}

\section{Journals}

Édition électronique

URL : http://journals.openedition.org/corela/1476

DOI : 10.4000/corela.1476

ISSN : 1638-573X

\section{Éditeur}

Cercle linguistique du Centre et de l'Ouest - CerLICO

\section{Référence électronique}

Anne-Marie Chabrolle-Cerretini, « La linguistique cognitive et Humboldt », Corela [En ligne], HS-6 | 2007, mis en ligne le 01 novembre 2007, consulté le 19 avril 2019. URL : http:// journals.openedition.org/corela/1476 ; DOI : 10.4000/corela.1476

Ce document a été généré automatiquement le 19 avril 2019

\section{(c) (i) (2)(2)}

Corela - cognition, représentation, langage est mis à disposition selon les termes de la licence Creative Commons Attribution - Pas d'Utilisation Commerciale - Partage dans les Mêmes Conditions 4.0 International. 


\title{
La linguistique cognitive et Humboldt
}

\author{
Anne-Marie Chabrolle-Cerretini
}

1 La mise en relation de la linguistique cognitive avec Humboldt semble aller de soi. En effet, quoi de plus attendu que le rapprochement entre :

- Humboldt qui fut le premier à conceptualiser, au début du XIX ${ }^{\mathrm{e}}$ siècle, les rapports entre le langage, la pensée et le monde, dans le cadre d'un programme de description structurale des langues, et

- la linguistique cognitive des $\mathrm{XX}^{\mathrm{e}}$ et début $\mathrm{XXI}^{\mathrm{e}}$ siècles ayant pour objet l'étude des liens entre le langage, l'esprit et le cerveau, décrits à partir du système des langues.

2 Si le propos est évident, son exécution s'avère d'une grande complexité car les liens ne sont pas toujours attestés et vérifiables

3 Dans un premier temps, il faut nous entendre sur ce que nous allons examiner côté « Humboldt » et côté « linguistique cognitive », ainsi que sur la nature des liens envisagés. Nous chercherons à relier :

- Les textes linguistiques de Humboldt, écrits en allemand et en français, avant sa mort en 1835, dans une Europe dominée par la Grammaire comparée qui, par la suite, ont été lu en langues originales ou en traduction, de façon souvent partielle, et

- des textes contemporains multilingues relevant d'un espace épistémologique en pleine ébullition qui jouissent par ailleurs de toute la modernité des connaissances, des moyens de communication et de diffusion qui accélèrent et façonnent à la fois, plus collectivement, une réflexion théorique. 

cognitive, le contenu théorique et l'antériorité des textes humboldtiens nous invitent à formuler les questions qui suivent : constituent-ils une base théorique à laquelle on s'alimente pour penser dans le cadre scientifique d'aujourd'hui la problématique du rapport entre la langue et la culture qui préoccupait déjà Humboldt? Peut-on atteSter une filiation des idées du linguiste allemand avec les théories développées dans les différents courants de la linguistique cognitive contemporaine? Et si oui, quelles sont ces idées d'une part, quels sont ces courants de l'autre?

6 Avant d'avancer, dans la seconde partie du présent article, quelques éléments de réponse à ces questions, qui contribueront à éclairer la problématique de ce numéro spécial de Corela, il me semble nécessaire de consacrer la première partie de ma contribution à un rapide état de l'édition, de la diffusion et donc de la réception de Humboldt dans les différentes aires linguistiques et culturelles, car ces données alimentent très directement notre propos.

\section{L'édition et la réception des idées linguistiques de Humboldt}

\subsection{0-1835}

7 La théorie du langage de Humboldt est issue d'un cheminement anthropologique. On le sait, à la fin du XVIII ${ }^{e}$ siècle, le concept d'homme, examiné jusqu'à cette époque à l'intérieur du discours philosophique, tend à s'autonomiser et à devenir l'objet d'une science nouvelle, l'anthropologie. Si Humboldt, dans ses premières réflexions, participe complètement de ce débat, il parcourt assez vite la distance intellectuelle qui sépare son essai sur l'anthropologie comparée élaboré au tournant du XIX ${ }^{e}$ siècle du plan d'une étude linguistique comparative qu'il présente en 1820. Dans sa recherche de ce qu'est l'humanité, Humboldt comprend que c'est cette faculté universelle qu'est le langage qui se spécifie dans les langues, parlées par tous les individus peuplant la terre, qui va lui permettre de parvenir à la caractérisation des hommes. Convaincu que la diversité des langues est l'indice d'une variété de pensée, il conçoit l'idée que pour cerner la diversité humaine, il lui faut appréhender l'ensemble des développements possibles de l'esprit humain que laisse entrevoir la multiplicité des langues.

8 L'originalité théorique tient à l'approche globale du langage. Il fonde une linguistique sur :

9 1. La reconnaissance de la diversité des langues. Ce qui n'était pas acquis d'avance car si l'Occident a toujours été plurilingue, cette situation n'a jamais constitué un fil conducteur de l'étude linguistique. Ainsi pour la première fois dans l'histoire occidentale de la description des langues se développe un intérêt scientifique pour la diversité des langues qui, loin de constituer un handicap pour l'humanité en est la richesse même.

10 2. La connaissance de la diversité des langues. Il entend la cerner à partir d'une définition de la langue dans des rapports de dépendance avec la pensée, l'homme et la nation, en prenant en compte ensemble et reliés entre eux tous les aspects de cette aptitude 
naturelle au langage. Pour la première fois on peut lire une théorisation de la fonction cognitive et sa mise en liens avec les aspects expressif, communicatif, historique, social du langage.

11 Du vivant de Humboldt, certains de ses textes en allemand ont été édités. Il s'agit en fait de ses discours prononcés à l'Académie de Berlin :

Über das vergleichende Sprachstudium in Beziehung auf die verschiedenen Epochen der Sprachentwicklung, [Sur l'étude comparée des langues dans son rapport aux différentes époques du développement du langage] lu le 29 juin 1820 , publié en 1822.

12 C'est la première exposition publique des idées linguistiques de Humboldt. Dans ce texte il est question d'une science linguistique autonome ayant pour objet les langues et qui ne serait plus subordonnée à l'histoire de l'homme. Il établit en effet un nouveau rapport entre l'homme et le langage. Sont développés, entre autres, les concepts de Innere Sprachform [forme interne], Verschiedenheit [diversité] et de Weltansicht [vision du monde].

Über die Aufgabe des Geschichschreibers, [La tâche de l'historien], lu le 12 avril 1821, publié en 1822.

13 Bien que consacré à l'écriture de l'histoire, ce texte peut se lire comme le versant méthodologique du premier discours.

Über das Entsteben der Grammatischen Formen, und ihren Einfluss auf die Ideenentwicklung, [Sur la naissance des formes grammaticales et leur influence sur le développement des idées] lu le 17 janvier 1822, publié en 1823.

Ce texte traite du lien entre les formes grammaticales de la langue et le développement des idées qu'elles permettent.

Über den Dualis, [Le duel] lu le 26 avril 1827, publié en 1830.

Ce texte est une mise en application des principes théoriques et méthodologiques du projet de recherche à partir du traitement de la présence éventuelle du duel dans les langues.

16 À ces textes s'ajoute la publication française en 1827 de l'échange épistolaire datant de 1826 avec le Français A. Rémusat autour de leur compréhension du chinois, Lettre à $M$. Abel-Rémusat sur la nature des formes grammaticales en général et sur le génie de la langue chinoise en particulier.

On remarquera que seuls les textes plutôt programmatiques sont publiés (celui sur le duel est peut-être l'exception). Toutes les études descriptives des langues amérindiennes, du Kavi, des langues dravidiennes, baltiques et malayo-polynésiennes demeurent des manuscrits. Les travaux sur le basque échappent à cette logique et sont publiés dès le début du XIXe siècle : il s'agit en 1812 d'un article pour le Deutsches Museum de F. Schlegel, en 1817 d'un autre pour le Mithridates d'Adelung et J.- S.Vater et en 1821 de Prüfung der Untersuchungen über die Urbewohner Spaniens vermittelst der Vaskischen Sprache. Dans ces trois textes, les principes d'une analyse interne sont développés au service de la recherche des premiers habitants de l'Europe par l'étude de la langue basque. La structure du basque est dégagée à partir de ses lois internes tant d'un point de vue phonétique et morphologique, que sémantique. La comparaison avec d'autres langues intervient dans les démonstrations ou à titre de contre-exemples. Le contexte particulier de recherche sur le basque a persuadé Humboldt de la nécessité d'un renouveau méthodologique dans l'étude des langues. Elle l'a mis aussi sur la voie d'une conception du langage comme possible objet d'étude à partir de l'examen de langues particulières. 

évidemment, mais aussi des pays où il vécut. Ses fonctions ministérielles et ses voyages personnels qui l'entraînent en Italie, dans toute l'Allemagne, en Suisse, en France, en Angleterre lui permettent d'entrer en relation avec nombre de personnalités marquantes des mondes intellectuel, politique et artistique de son époque. Ses prises de position sur des sujets d'actualité comme la généalogie des langues et la classification, ou sur ses projets d'une linguistique générale sont des occasions qui ont facilité la diffusion de ses idées qui circulent ainsi déjà de son vivant. sont les échanges entre les Américains et les Européens (en fait essentiellement les Français et les Allemands). Les idées européennes via les Idéologues, Volney, Du Ponceau, les frères Humboldt vont être assimilées. Outre-Atlantique la scission entre les recherches sur les langues indiennes et l'anglais-américain ne s'est pas encore produite. continents, des voyageurs, des explorateurs et des missionnaires rapportent des informations sur des langues encore inconnues en Europe. Les archives européennes se remplissent de documents sur un nombre toujours croissant de langues et accumulent aussi davantage de références sur une même langue. Vocabulaires, dictionnaires, grammaires et récits de voyages avec lexique d'appoint vont permettre la découverte de langues finno-ougriennes, sémitiques, malayo-polynésiennes, amérindiennes, africaines, du chinois et aussi le déchiffrement de l'écriture égyptienne. Ce qui est sûr c'est que les frères Humboldt ont largement contribué à cet enrichissement linguistique et aux débuts des contacts scientifiques concernant la linguistique entre l'Amérique du Nord et l'Europe. Alexander, le géographe, rapporte d'Amérique des grammaires et des lexiques de missionnaires espagnols qui s'étaient employés à consigner des descriptions de langues amérindiennes lors de la conquête du nouveau continent. On ne peut sousestimer le fait qu'Alexander au début du XIX ${ }^{e}$ siècle, à Paris, présente devant l'Assemblée, plusieurs fois par semaine, ses travaux et surtout expose sa vision dynamique de la nature, sa théorie des liens existants entre tous les éléments sur terre qui rendent possible son équilibre général. Plus connu, plus mondain aussi, c'est bien Alexander qui a le plus grand projet éditorial de l'époque, Relation historique du voyage aux régions équinoxiales $d u$ Nouveau Continent qui réunissait des physiciens, mathématiciens, dessinateurs, graveurs et cartographes et auquel Wilhelm est associé pour la rédaction de la partie linguistique. Ce dernier apportera une contribution plus modeste que prévue. Néanmoins, le texte Essai sur les langues du Nouveau Continent écrit en 1812 directement en français pour le livre d'Alexander, qui n'a d'étude sur les langues d'Amérique que le nom, contient des vues théoriques et programmatiques de premier ordre sur l'étude des langues telle que la conçoit Wilhelm. Enfin, il est nécessaire d'évoquer l'harmonie intellectuelle règnant entre les deux frères Humboldt. S'ils n'ont pas les mêmes passions, les mêmes objets d'étude, il y a un terrain d'entente sur les façons de les envisager. Que ce soit dans Relation historique du voyage aux régions équinoxiales du Nouveau Continent ou dans Sur l'étude comparée des langues dans son rapport aux différentes époques du développement $d u$ langage, nous découvrons cette même approche systémique de la nature et des langues ainsi qu'un souci identique d'allier l'observation et la théorie.

Wilhelm ne sera pas uniquement un passeur de l'exceptionnel corpus de son frère, mais il traite aussi lui-même ces documents. Il rédige des grammaires, des dictionnaires, et décrit de nombreux aspects structurels des langues d'Amérique du Nord. J. Pinkering, 
spécialiste du cherokee, de l'iroquois, entre autres langues, qui publie l'ouvrage de J. Heckewelder en 1819 sur les nations indiennes, An account of the History, Manners and Customs of the Indian Nations, who once inhabited Pennsylvania and the Neighbouring States va correspondre avec Humboldt.

La linguistique de Humboldt, de son vivant, n'est pas très suivie, mais elle voyage. Pour reprendre le terme employé par D. Di Cesare ${ }^{2}$, Humboldt est frappé d'« inactualité » parce que la compréhension de la diversité linguistique a perdu de son attractivité et que les questions sur l'essence du langage, les rapports entre la nation et la langue ont été débattues largement au XVIII ${ }^{e}$ siècle. L'objet d'Une étude comparée des langues ne correspond plus à l'attente des années vingt du $\mathrm{XIX}^{\mathrm{e}}$ siècle. Du point de vue méthodologique, l'abîme se révèle tout aussi conséquent. Alors que la Grammaire comparée, sur le modèle de la biologie notamment, peut présenter la caution de «scientificité » ardemment recherchée, le projet humboldtien est rapidement taxé de "philosophique», ce qui signifiait implicitement qu'il était inapplicable et qu'il ne répondait pas (selon les tenants de la Grammaire comparée bien sûr, ce qui n'est pas complètement juste) aux critères empiriques incontournables mis en avant par une tendance positiviste renforcée.

En Europe, au XIX siècle, l'intérêt porté à la linguistique de la diversité est limité. Il n'est pas très tourné non plus vers les langues non indo-européennes. La Grammaire comparée liée à ses débuts, de par ses motivations, aux langues de l'Inde et du continent européen, porte un regard, de fait, plus distant sur les autres langues. Comme l'ont fait remarquer successivement $\mathrm{S}$. Auroux ${ }^{3}$ et J. Trabant ${ }^{4}$, les questions laissées pour compte se connectent souvent à l'étude de langues marginalisées et c'est ainsi que le projet de Humboldt va constituer le «réservoir des linguistiques oppositionnelles» que l'on peut relier sûrement aux études de langues non indo-européennes. Cette hypothèse semble se vérifier aux Etats-Unis au début du XIX siècle où l'influence de Humboldt est attestée chez les linguistes qui entreprennent effectivement la description des langues amérindiennes comme J. Pickering. Ce sera encore vrai, me semble-t-il, à la fin du XIX ${ }^{e}$ siècle - début du XXe siècle. Il faudra compter alors avec des linguistes influencés par les textes de Humboldt comme D. G. Brinton, son traducteur américain qui avait étudié à Heidelberg, Paris et Vienne, connaissait bien les textes du linguiste pour lesquels il ne cachait pas son empathie, travaillait sur la classification des langues amérindiennes et traduisit en 1885 un manuscrit de Humboldt sur les structures verbales des langues indiennes du nord de l'Amérique ${ }^{5}$. On peut citer également F. Boas (eskimo), E. Sapir (eskimo) et B. L. Whorf (langues amérindiennes et en particulier le hopi) qui contribuèrent au fondement de l'ethnolinguistique. En Europe, la remarque de S. Auroux et J. Trabant peut s'appliquer à J. H. Steinthal (langues africaines), J. C. E. Buschmann (langues malayo-polynésiennes), L. Adam (langues amérindiennes), J. Baudoin de Courtenay (lituanien - langues finno-ougriennes) puis L. Tesnière (langues finnoougriennes).

\subsection{Après 1835}

Après la mort de Humboldt, l'édition des textes est cahotique, mais c'est surtout le réel début d'une diffusion des textes dans d'autres langues. On note dès 1859 la traduction en français de De l'origine des formes grammaticales et de leur influence sur le développement des idées et une traduction en russe ${ }^{6}$ de l'Introduction à l'œuvre sur le Kavi. 

l'Académie à partir de 1903, les textes de linguistique générale se répartissant pour la plupart dans les volumes quatre à sept. Ensuite, l'histoire ${ }^{7}$ des manuscrits légués par le linguiste en 1835 à la Bibliothèque Royale de Berlin se confond en partie avec l'histoire de l'Allemagne. La plupart des descriptions de langues ont disparu et réapparaîtront partiellement en 1976 à la bibliothèque Yaguellone de Cracovie grâce aux recherches de K. Mueller-Volmer. notamment aux Etats-Unis et en Europe de l'Est. Les linguistes sont encore à cette époque souvent des germanistes et un grand nombre est aussi d'origine allemande. Humboldt a été lu, par ailleurs, beaucoup, en traduction à partir des années 1970. D'une façon générale, il est possible de dire que si l'on fait abstraction des œuvres sur le basque qui ont été traduites dès le XIX ${ }^{e}$ siècle en espagnol, c'est à partir effectivement du dernier quart du XXe siècle que l'Introduction à l'œuvre sur le Kavi et le discours de 1820 sont traduits dans les langues de grande diffusion. Pour ne citer que les traductions anglaises, françaises, italiennes et espagnoles à partir de 1970, des textes dont il a été question jusqu'à présent:

En anglais :

Aarsleff H., On language. The Diversity of Human Language-Structure and its Influence on the Mental Development of Manking, Edited by, Trans. Peter Heath, Cambridge, Cambridge University Press, 1988.

Harden T., Farelly D., Wilhelm von Humboldt Essays on language, Edited by, Trans. John Wieczorek and Ian Roe, Peter Lang, 1997.

\section{En français :}

Caussat Pierre, Introduction à l'oeuvre sur le Kavi et autres essais, Paris, Seuil, 1974. Laks André, Disselkamp Annette, G. de Humboldt. La tâche de l'historien, Villeneuve-d'Ascq, Presses Universitaires de Lille, 1985.

Caussat Pierre, La langue source de la nation, Sprimont, Mardaga, 1996.

Caussat Pierre, Patrimoine Littéraire européen, Anthologie en langue française sous la direction de Jean-Claude Polet Volume 11a, Renaissances nationales et conscience universelle, Paris, Bruxelles, De Boeck Université, 1999.

Rousseau Jean, Thouard Denis, Lettres édifiantes et curieuses sur la langue chinoise. Humboldt-Abel Remusat, Villeneuve-d'Ascq, Editions du Septentrion, 1999.

Thouard Denis, Sur le caractère national des langues, Paris, Seuil, Points Essais, 2000.

Samain Didier, "Annonce d'un essai sur la langue et la nation basque avec indication du point de vue et du contenu de l'ouvrage ", Verbum XXVII 1-2, Wilhelm von Humboldt, les langues et sa théorie du langage, numéro coordonné par A-M Chabrolle-Cerretini, Nancy, Presses Universitaires de Nancy, 2005.

\section{En italien:}

Di Cesare Donatella, Humboldt, La diversità delle lingue, Roma-Bari, Biblioteca Universale Laterza, Editori Laterza, 1993.

\section{En espagnol:}

Sobre la diversidad del lenguaje humano y su influencia sobre el desarrollo espiritual de la humanidad, Trad. de Ana Agud. Barcelona, Anthropos Editorial del hombre, 1990, 
Escritos sobre el lenguaje, Trad. de Andrés Sánchez Pascual, Ripollet, Ediciones Península, 1991. celle de l'étude des langues amérindiennes. Cette dernière va se spécifier toujours davantage sur des questions de relativité linguistique et fonder l'ethnolinguistique. E. Sapir, B.L. Whorf et F. Boas y participent, mais meurent à quelques années d'intervalle, soit respectivement, 1939, 1941 et 1942. Ces trois éminents linguistes peuvent constituer dans des proportions variables un relais de la pensée humboldtienne. Il faut dire que F. Boas, né en Allemagne en 1858 a une culture profondément germanique même s'il s'en défendait. Il est géographe de formation et cite abondamment A. von Humboldt, plus que Wilhelm. Il rejoint définitivement les Etats-Unis en 1886 et contribue à l'autonomisation de l'ethnolinguistique en travaillant à la reconstitution de l'histoire des sociétés amérindiennes. Étudiant de F. Boas, E. Sapir sera l'enseignant de B.L. Whorf.

Aux Etats-Unis, la première moitié du $\mathrm{XX}^{\mathrm{e}}$ siècle est également associée à l'origine des sciences cognitives $^{8}$ que l'on peut faire remonter aux conférences Macy, du nom de la fondation qui finance les actions en faveur de la santé mentale. Le monde vient d'être ébranlé par la seconde guerre mondiale et les États-Unis sont traumatisés par l'idée que les guerres se fomentent d'abord dans les esprits humains. Ces conférences pluridisciplinaires qui réunissent régulièrement dès 1946 des mathématiciens, logiciens ingénieurs, physiologistes, économistes, anthropologues, neurophysiologistes et psychologues ont pour ambition d'édifier une science générale du fonctionnement du cerveau qui contribuera à la régulation de la paix mondiale. Les travaux de F. Boas sont convoqués ainsi que ceux de ses épigones. En effet, F. Boas avait pris sur la fin de sa vie des directions qualifiées davantage de «culturalistes" par la communauté des ethnolinguistes, traitant de questions plus d'actualité comme l'éducation et le nationalisme, et c'est à ce titre que des anthropologues comme R. Benedict, son assistante, ou G. Bateson participeront à ces discussions. Sous la pression aussi de la nouveauté informatique, l'esprit est assimilé à une machine, puis le cerveau pressenti comme un réseau d'interconnexions. Le programme initial consiste ainsi à rendre compte de la cognition humaine de façon modélisée. De cette période scientifique américaine est issue la linguistique cognitive que $C$. Fuchs définit aujourd'hui ainsi :

Sous la dénomination de "linguistique cognitive", on s'accorde en effet à regrouper un ensemble de courants qui partagent - par-delà leurs différences - un objectif commun: celui de proposer des théories de la langue qui soient non seulement opératoires et générales, mais également susceptibles de s'articuler de 
façon explicite avec des modèles généraux de l'architecture fonctionnelle de l'esprit

et/ou de l'architecture neuronale du cerveau. ${ }^{9}$ questions de démarches scientifiques et des postulats cognitifs fondamentaux :

\footnotetext{
- Le premier, appelé « cognitiviste » ou « computo-représentationnel symbolique » qui produit les grammaires formelles de N. Chomsky. La démarche est hypothético-déductive. L'approche du langage est modulariste. Une grande place est accordée à la syntaxe. La modélisation est d'ordre logico-algébrique.
}
- Le second, nommé « constructiviste » qui a conduit aux grammaires cognitives avec R. Langaker ${ }^{10}$. La démarche est au contraire inductive, d'où un recours possible à un corpus textuel, exclu dans le premier courant. Il n'y a pas de spécificité du module langue. Le postulat est celui de mécanismes cognitifs généraux. La syntaxe est traitée dans ses rapports avec la sémantique. La modélisation a recourt à la géométrie et rend compte de l'approche interactionniste de la langue.

Depuis, la réflexion théorique a progressé. Elle a aussi intégré11 un courant né en Europe et s'est enrichie de la nécessaire discussion avec toutes les disciplines impliquées dans les sciences cognitives. Les différentes approches tant américaines qu'européennes ont fait éclater cette bipolarisation et se situent désormais sur un continuum. Après ce synthétique exposé des grandes caractéristiques de la réception de la linguistique humboldtienne, on comprend qu'il était attendu de trouver le linguiste référencé dans les textes critiques relevant de la linguistique cognitive.

Curieusement, on trouve des mentions à Humboldt dans les deux courants historiques de la linguistique cognitive qui se définissent de façon très différente sur la question de la diversité des langues, mais aussi, à divers titres, dans bon nombre des multiples tendances théoriques actuelles. "Petits arrangements " avec Humboldt, lectures partielles de sa théorie du langage, il est maintenant temps d'apporter quelques éclairages sur cette situation.

\section{Les textes humboldtiens et la linguistique cognitive : source, filiations et idées débattues}

\subsection{Les filiations discutées}

\subsubsection{N. Chomsky}

Ma première remarque concerne la pertinence et l'intérêt d'envisager Humboldt comme l'une des sources de la linguistique cognitive. On l'a vu dans la première partie, les idées de Humboldt sont connues dès le début du XIX ${ }^{\mathrm{e}}$ siècle aux Etats-Unis comme en Europe. Elles ont nourri, de façon attestée, l'ethnolinguistique et l'étude des langues amérindiennes. Elles ont alimenté la linguistique russe par le biais d'A. Potebnja ${ }^{12}$, entre autres. En Europe de l'Est ${ }^{13}$, plus globalement, P. Sériot ${ }^{14} n^{\prime}$ hésite pas à parler de néohumboldtianisme pour désigner des positions théoriques qui s'appuyent principalement sur les concepts ayant un rapport avec la formalisation des liens entre la langue, la 
pensée et la nation. On peut déduire aussi de la première partie que les textes humboldtiens participent de façon plus inconsciente à ce «background » théorique dont émergera la linguistique contemporaine et la linguistique cognitive des deux côtés de l'Atlantique.

Ainsi, s'il est approprié d'évoquer Humboldt, dans les limites que je viens de rappeler, comme l'une des sources de la linguistique cognitive contemporaine, l'intérêt est relatif car à être partout, il risque bien d'être dissous dans ce fonds linguistique commun où se retrouvent d'autres auteurs dont la réception est également soumise à des conditions culturelles, politiques, sociales externes à la discussion linguistique même.

Il me semble plus intéressant de mentionner, en contrepoint, que Humboldt est parfois convoqué comme source, de façon incorrecte. Le premier exemple est celui de $\mathrm{N}$. Chomsky qui se rapporte à Humboldt pour des besoins de légitimité scientifique et historique. Le linguiste américain s'est créé une continuité historique, de Port-Royal à luimême, en passant par Humboldt. H. Meschonnic ${ }^{15}$ a bien démontré comment $\mathrm{N}$. Chomsky tire à lui Humboldt par une interprétation erronée car ne portant pas sur la totalité du texte, en le situant, de plus, dans une opposition à Saussure qui est discutable car l'objet des « deux linguistiques » était différent, mais pas forcément antagoniste ${ }^{16}$.

La traduction des textes joue ici un rôle important. L'écriture de Humboldt a au moins deux caractéristiques : elle n'est pas terminologique et doit être appréhendée comme une pratique de sa théorie du langage. Quand le linguiste défend que la langue n'est pas un ouvrage terminé (ergon), mais une dynamique (energeia), quelque chose en train de se faire continuellement, il est logique que son texte soit la formulation incessante d'une pensée et qu'il n'arrête jamais une terminologie. Par ailleurs, comme corollaire de sa définition de l'activité cognitive dépendante dans sa dernière étape de la fonction de communication, les plans de la langue et du discours ne sont jamais dissociés. Le discours est la finalité de la langue. C'est dans l'actualisation que la langue exprime ses potentialités. Rien d'étonnant, en ce cas, que dans ses propos sur la traduction il insiste sur le fait que l'on traduit un texte et non une langue et que l'on traduit un travail et non une œuvre. Il résulte qu'un concept n'est nulle part défini en bonne et due forme, mais que son contenu se constitue au fil des textes. C'est en définitive, le lecteur de Humboldt qui est en mesure de réunir les différents traits définitoires dispersés et de proposer une définition.

La traduction incriminée concerne celle des idées relatives au fonctionnement du langage. Elle est analysée ainsi par H. Meschonnic :

La générativisation de Humboldt s'opère par un double effet de traduction qui n'est pas sans portée. Ainsi pour le terme erzeugen, traduit par «to generate" (engendrer) avec toute apparence de raison. Mais il motive vers une production et un engendrement réel, biologique, ce qui n'était censé avoir qu'un sens logique. Il se fait une métaphorisation qui contribue à une confusion sur la créativité. [...] Mais le bénéfice est considérable : il fournit la continuité d'une longue tradition, d'autant plus unie si Port-Royal se continue dans Humboldt - le cumul de posséder à la fois la tradition et l'invention, la vérité et son histoire, identification à effet charismatique. La formalisation y ajouté l'ordre et la certitude scientifiques. ${ }^{17}$

Quand on sait combien les notions centrales chez N. Chomsky de compétence et de performance se trouvent liées à la question de la créativité, il y a de quoi être troublé par de tels raccourcis historiques. 
Dans Language and Mind (1968) voici un exemple de la manière dont s'opèrent les appropriations :

La structure profonde est liée à la structure superficielle par certaines opérations mentales - selon la terminologie moderne, par des transformations grammaticales. Chaque langue peut être considérée comme une relation particulière entre le son et le sens. En poussant la théorie de Port-Royal jusqu'à ses conclusions logiques, il faut considérer la grammaire d'une langue comme un système de règles qui caractérise les structures profonde et superficielle et leurs relations transformationnelles et qui - pour rendre compte de l'aspect créateur de l'utilisateur du langage - les caractérise dans un domaine infini de structures superficielles et profondes couplées. Pour emprunter la terminologie qu'utilisait Wilhelm von Humboldt dans les années 1830, le locuteur fait un usage infini de moyens finis. Sa grammaire doit donc contenir un système fini de règles qui engendre une infinité de structures profondes et superficielles liées de façon appropriée; elle doit aussi contenir des règles qui relient ces structures abstraites à certaines représentations du son et du sens $[\ldots . . .]^{18}$

On notera plus récemment que N. Chomsky a pris de la distance par rapport à Humboldt, le reléguant au chapitre des textes imprécis et dépassés :

La « révolution cognitive » a renouvelé et reformulé de nombreux aperçus, acquis et dilemmes de ce que l'on pourrait appeler le « première révolution cognitive » des $\mathrm{XVII}^{\mathrm{e}}$ et $\mathrm{XVIII}^{\mathrm{e}}$ siècles, elle-même inséparable de la révolution scientifique qui a transformé si radicalement notre compréhension du monde. On s'aperçut à cette époque que le langage met en jeu «l'usage infini de moyens fini », pour reprendre l'expression de Wilhelm von Humboldt; mais cette intuition ne pouvait être développée que de façon limitée, car les idées sur lesquelles elle reposait demeuraient vagues et obscures. Au milieu du $\mathrm{XX}^{e}$ siècle, les progrès accomplis dans les sciences formelles ayant fourni des concepts adéquats sous une forme nette et claire, il devint possible de donner une explication précise des principes computationnels qui engendrent les expressions d'une langue et ainsi de se représenter, du moins partiellement, l'idée d'un " usage infini de moyens finis »" pour les principes générativistes, cette opposition est peut-être un peu simpliste. On prélève une phrase d'un long développement qui, avec les moyens du XIX ${ }^{\mathrm{e}}$ siècle, théorise l'interdépendance procréatrice entre la langue, la pensée, la réalité et la nation et l'on parle d'obscurité. Il se trouve surtout que la linguistique de la diversité n'a guère d'affinités avec le programme générativiste. Sur ce point précis, les « moyens finis » sont à considérer dans une approche synchronique car la forme interne qui est l'essence de l'individualité de la langue ne cesse de proposer dans la langue de nouveaux moyens.

\subsubsection{L'« hypothèse Sapir et Whorf »}

Le second exemple de filiation problématique qui mérite d'être abordé ${ }^{20}$ est celui de la célèbre « Hypothèse Sapir et Whorf ». Cette expression si abondamment reprise n'est du fait ni de Sapir ni de Whorf, mais de J.B. Carroll ${ }^{21}$ qui s'est chargé en 1955 (Language, Thought and Reality, 1956) de réunir et d'éditer des textes que Whorf projetait de publier en mémoire à E. Sapir et A. Fabre d'olivet.

49 L'expression a eu le succès que l'on sait sans avoir eu jamais une réelle définition et focalisant sur elle, au contraire, des interprétations très hétérogènes. Participant peutêtre de cette même nécessité de faire converger un certain nombre d'intuitions, d'hypothèses, ce ne sont pas les auteurs eux-mêmes, mais la communauté des linguistes qui a mis en relation les idées des deux Américains avec celles de Humboldt. Les 
rapprochements se font dans des configurations comme «langue et culture », « langue et pensée », "relativité linguistique » et " déterminisme linguistique ». Ce qui est mis en relation explicitement ou non est le concept humboldtien de «vision du monde [Weltansicht] ${ }^{22}$ " avec l'idée que la langue structure l'expérience que l'homme a du monde. La vision du monde est au cœur de la théorie du langage et désigne la saisie organisée du monde extérieur par la langue, la façon de comprendre la réalité extralinguistique, la manière de s'y référer et de la construire par le discours. Ce concept est directement lié à l'activité cognitive qui est chez Humboldt «un acte de représentation du donné sensible qui est orienté, ordonné en fonction d'une règle ${ }^{23}{ }_{\eta}$. Cet "acte de représentation» est une opération de synthèse qui se décompose en quatre étapes qui doivent se réaliser pour que le processus du langage s'accomplisse. La première étape est celle de la représentation de l'objet par l'esprit, la perception subjective de celui-ci, puis vient la phase de la représentation proprement dite, puis celle de la réalité acquise de cette représentation dès lors que l'individu entre en communication avec autrui et réfère à cette représentation et enfin la dernière qui estla réalité obtenue dans la réciprocité quand autrui la reproduit. Si Humboldt distingue différentes phases dans cette activité complexe de synthèse qui va de la représentation du monde en passant par l'épreuve sur autrui de la pensée jusqu'à un accord sur celle-ci, il est évident qu'à ses yeux, c'est un tout qui doit être appréhendé.

Les textes de Humboldt, Sapir et Whorf ont subi des torsions pour qu'ils se rencontrent sur la formalisation de ce rapport entre la langue, la pensée et le monde.

Commençons par E. Sapir qui s'est toujours exprimé avec réserve sur le principe d'immanence qui prévaut au début $\mathrm{du} \mathrm{XX}^{\mathrm{e}}$ siècle dans l'approche du langage, et qui, appliqué strictement, conduit, selon lui, à une étude qu'il juge faussée, renvoyant les dimensions anthropologique, sociologique, ethnologique et psychologique à la périphérie d'une étude de la structure de la langue. Sensibilisé au fait que le langage est lié à la construction socioculturelle et psychologique de l'individu, E. Sapir considère que l'étude du langage ne peut être qu'interdisciplinaire. Il va chercher à définir ce qui fait de la langue un outil de communication et de socialisation et posera rapidement une fonction symbolique qui peut s'apparenter à la fonction de représentation de Humboldt :

[...] ne faut-il pas voir [...] la preuve que le langage a le pouvoir d'analyser les données de l'expérience en éléments théoriquement dissociables et d'opérer, dans des proportions diverses, cette fusion du virtuel et du réel qui permet aux humains de transcender ce qui est immédiatement donné dans leur expérience individuelle et d'accéder à un domaine commun ?24

La définition d'une fonction symbolique du langage conduit E. Sapir à revisiter avec prudence la nature des liens entre une langue et une culture.

Cette attitude à peser ses propos se vérifie au moins sur deux plans :

- Le développement coordonné du linguistique et du culturel,

- Les éléments de la langue (lexique et grammaire) concernés par ce rapport.

Ses études sur les langues et les cultures indiennes, en comparaison avec celles de l'Europe, ont amené E. Sapir à la thèse que la langue et l'activité culturelle ne sont pas soumises directement aux mêmes influences et évoluent à des rythmes différents. À un 
stade primitif de la construction de la langue, un parallèle entre la culture et la langue peut, par contre, s'envisager au vu de la nature symbolique de cette dernière :

Le fait que le langage soit une parfaite traduction symbolique des données de l'expérience, le fait qu'il ne puisse dans la pratique être séparé de l'action et qu'il soit le porteur d'une infinité de nuances expressives sont autant de caractéristiques psychologiques universellement valables. ${ }^{25}$

Pourtant, en dépit de la propriété de la langue à construire et transmettre l'expérience humaine, la correspondance directe entre la forme de la langue et la culture ne peut être postulée :

La tendance de certains sociologues et ethnologues à voir dans les catégories linguistiques une expression directe de quelques-uns des aspects les plus manifestes d'une culture doit être combattue comme contraire à ce que révèle l'examen des faits. [...] La signification culturelle de la forme linguistique ne doit pas être cherchée au niveau des caractéristiques manifestes d'une culture donnée mais à un niveau bien plus profond. ${ }^{26}$

57 C'est à un niveau linguistique plus profond qu'il faut chercher la structuration de l'expérience, qui sera sans doute moins réactif, ou moins rapidement réactif, aux changements des comportements culturels.

E. Sapir va finalement restreindre au seul lexique la possibilité d'une mise en relation.

B. L. Whorf, quant à lui, va travailler à éclaircir ce processus cognitif qui organise les données de l'expérience humaine.

Pour B. L. Whorf, chaque langue est :

[...] un vaste système de structures différent des autres, dans lequel il existe un ordonnancement culturel des formes et des catégories qui non seulement permet à l'individu de communiquer, mais également analyse le réel, remarque ou néglige des types de relations et de phénomènes, canalise son raisonnement et jalonne peu à peu le champ de sa conscience. ${ }^{27}$

61 Il posera un principe de "relativité linguistique » qu'il appliquera à l'ensemble de la langue, lexique et structure grammaticale. Le principe de relativité que Whorf est le seul a avoir arrêté est ainsi défini :

On aboutit ainsi à ce que j'ai appelé le "principe de relativité linguistique», en vertu duquel les utilisateurs de grammaires notablement différentes sont amenés à des évaluations et à des types d'observations différents de faits extérieurement similaires, et par conséquent ne sont pas équivalents en tant qu'observateurs, mais doivent arriver à des visions du monde quelque peu dissemblables..$^{28}$

62 Sur le même principe que celui de la relativité en physique qui veut que la position d'un individu dans la nature détermine la vue qu'il en a, un locuteur est observateur de la nature commune, à partir de sa langue qui ne peut proposer qu'un découpage particulier de celle-ci, en fonction de la langue même. Ces données de l'expérience structurées seront alors conceptualisées de façon différente en fonction des structures linguistiques ellesmêmes, mais aussi :

[...] de la valeur analogique et suggestive de ces structures (par exemple, notre « espace imaginaire » et ses implications éloignées), et toutes les interpénétrations entre la langue et la culture - données constituant un ensemble qui pour une bonne part n'est pas de nature linguistique mais montre cependant l'influence structurelle de la langue. ${ }^{29}$

B.L. Whorf est parvenu à poser de telles corrélations à partir de ses activités professionnelles. Il est ingénieur chimiste employé dans une compagnie d'assurance 
contre les incendies quand il devient l'assistant d'E. Sapir à l'Université de Yale. Ses interventions sur des lieux sinistrés et la rédaction de constats avec les personnes impliquées l'ont mis sur la voie de l'hypothèse qu'il n'y avait pas que des causes purement matérielles, mais un facteur de "signification que revêtait cet état de fait aux yeux des gens [et] constituait parfois un des éléments ayant présidé au sinistre ${ }^{30}$ ». Il reprenait ainsi à son compte le postulat d'un rapport entre une structuration mentale et la langue. C'est l'exemple relaté d'un incendie dans une tannerie qui, selon lui, s'explique par une appréhension inadaptée de la situation nouvelle due à une représentation inexacte d'un objet, en l'occurrence, un ventilateur :

Dans un local destiné au séchage des peaux, on avait installé un ventilateur à une des extrémités pour produire un courant d'air à travers la salle ; à l'autre extrémité on avait aménagé une ouverture communiquant avec l'extérieur. Le feu se déclara à un des supports du ventilateur, lequel chassa les flammes en direction des peaux et attisa l'incendie, qui détruisit tout le stock. Cette situation dangereuse est déterminée tout naturellement par le terme "ventilateur", et son équivalent linguistique «quelque chose qui souffle " impliquant que sa fonction est obligatoirement de «souffler». Ce qui se traduit donc par «souffler de l'air pour sécher » et on oublie ainsi que l'appareil peut projeter d'autres choses, par exemple des flammes et des étincelles. ${ }^{31}$

La structuration de l'expérience s'opère à deux niveaux :

- Un niveau inférieur, qui est celui du champ perceptif. C'est le plan des « formes », soit l'organisation de la nature et celui des « noms », soit la mise de mots sur l'expérience que l'on a de la nature que l'on fragmente. Ce découpage de l'expérience en « isolats d'expérience ${ }^{32}$ » est lié à chaque langue.

- Un niveau supérieur, qui, lui, n'a pas de référence à une forme visuelle ou spatiale, s'organisent, à partir du plan inférieur, les unités, selon des règles combinatoires. C'est à ce niveau que l'on observe la manière dont se construit le sens dans les phrases. Ces façons ne sont pas « [...] assimilables aux phrases elle-mêmes, mais aux schémas des phrases et à la structure qui les caractérise. $»^{33}$

Le plan d'une recherche sémasiologique ${ }^{34}$ qu'il propose embrasse tous les aspects phonologique, morphologique, lexical et syntaxique de la structure linguistique. Il faut que l'analyse soit la plus complète possible et il convient aussi de savoir comment les locuteurs se servent de la structure. Dans cette perspective, Whorf envisage une analyse sur le plan discursif qui est une étude des variations intralangue dans différents genrestextuels. Il y a ainsi des caractérisations de style de conversation, de narration, de chanson, d'incantation, etc. Ce programme d'étude se caractérise par son exhaustivité et le fait que l'analyse est conçue jusqu'aux unités minimales de la langue, phonème, morphème et sème.

B. L. Whorf a pensé son travail sous forme de monographies. Son enquête sur les indiens Hopis vivant dans le désert de l'Arizona, éleveurs de moutons et agriculteurs malgré une terre infertile en est un exemple qui l'a conduit à mettre son hypothèse à l'épreuve de la structure grammaticale d'une langue. À partir de l'examen du système verbal qui ne marque pas la temporalité comme dans la plupart des langues du groupe indo-européen, B. L. Whorf a défendu l'idée que les Hopis avaient une conception particulière de l'espace, 
du temps et surtout de l'événement, marquée par une approche binaire du monde dans ses moindres sous-divisions et par une représentation métaphysique de celui-ci.

Même si, en 1939, c'est-à-dire presque à la fin de sa vie, il avait commencé une comparaison entre des langues amérindiennes et des langues de l'Europe de l'Ouest, ses textes ne laissent pas penser à une quelconque étude générale alimentée par des descriptions de langues dans leur rapport au monde.

Le sociolinguiste H. Gipper ${ }^{35}$, est le premier, dans les années soixante-dix, à reprendre le travail laissé par Whorf, pour vérification, impressionné à la fois par la témérité de ses propos et l'absence de contre-analyse de la part de ses détracteurs pour conclure à la validité du postulat, même si l'analyse linguistique sur la langue hopi est défectueuse.

Critiqué, puis validé dans ses hypothèses, relu avec attention depuis une dizaine d'années partout dans le monde, B. L. Whorf contribue à relancer la discussion autour du concept de vision du monde. À bien y regarder, Humboldt ne peut être qu'une source lointaine pour Whorf. Je dirais que les deux hommes partagent simplement une interrogation sur le rapport entre la structure linguistique et la représentation par la langue de la réalité. Les derniers travaux de J. Joseph vont dans ce sens et considèrent que les idées de Whorf sont bien davantage influencées par celle du sémanticien A. Korzybski et plus encore par celles de la théosophie ${ }^{36}$, doctrine morale qui l'a accompagné depuis son enfance et dans sa carrière professionnelle jusqu'à sa mort.

Un point pourtant rapproche Humboldt et Whorf. Il s'agit de l'interprétation fausse de leurs idées, particulièrement celle du déterminisme excessif que l'on associe à la vision $d u$ monde comme à l' « Hypothèse Sapir et Whorf " parfois réduite à la formule «une langue = une culture ».

Du côté des Américains, la thèse du déterminisme ne semble pas être alimentée par les deux linguistes. Ceux-ci ont été plutôt prudents, mais fermes dans ce qu'ils étaient en mesure d'affirmer sur le rapport même. Nulle trace de déterminisme dans leurs textes, il n'est question que de relativité. L'activité conceptuelle n'intervient qu'en second lieu, sur des données d'expériences déjà structurées. Il y a donc un premier niveau où l'expérience de la réalité est partagée par toutes les communautés.

Chez Humboldt, la définition de la langue liée de façon circulaire à la pensée, une nation et la vision du monde empêche de parler de déterminisme ou de conditionnement. La vision $d u$ monde n'est qu'une saisie particulière du monde par la langue, indispensable et inhérente à l'homme. Elle n'est en aucun cas une Weltanschauung, une conception du monde, un discours, un cadre de pensée organisé et rigide sur le monde.

\subsection{La diversité des langues débattue}

Les deux grands courants historiques de la linguistique cognitive abordent la diversité des langues différemment et pourtant chacun convoque Humboldt. On l'a vu du côté des cognitivistes et de N. Chomsky. Pour eux, l'analyse de la diversité est une donnée négligeable puisque c'est la part des invariants qui mobilise toute leur attention. Du côté des grammaires cognitives, le discours s'inverse, la référence à Humboldt se fait indirectement en posant le principe d'une "relativité linguistique » et l'analyse de la diversité par le biais de l'étude des représentations construites dans les langues est totalement investie. 
74 La question de la diversité n'est pas posée en ces termes par Humboldt. Ce dernier ne renvoie pas dos-à-dos l'universalité du langage et la particularité des langues. Il ne considère pas, surtout, les deux comme le recto/verso d'une même problématique.

Dans sa théorie du langage, Humboldt a toujours pensé le plan de l'universel et celui du particulier ensemble, sans doute parce que sa théorie est bien fondée sur l'objectivation de la question de la diversité.

Tout commence avec son explication de la diversité des langues comme le résultat de la dynamique de l'humanité. En effet, pour Humboldt, le langage s'est développé, en une seule fois, dans le cerveau humain lorsque celui-ci présenta une maturité suffisante pour l'utiliser.

77 Si l'origine du langage n'est pas un phénomène progressif, de même, celui-ci ne s'est pas développé dans le but de répondre aux besoins de communiquer de l'homme, mais parce qu'il s'est révélé indispensable à sa croissance intellectuelle, lui permettant d'appréhender le monde extérieur. Humboldt a inversé la relation causale habituellement avancée. C'est le langage qui génère un besoin de communication et non le contraire. Cette relation d'engendrement permet à Humboldt de concevoir la diversité des langues comme une manifestation de l'humanité qui se répète autant de fois qu'il y a de langues. Dans le même temps, il considère que cette individuation de la langue d'une aptitude partagée par les hommes le met dans la nécessité de ne jamais dissocier le plan du particulier du plan général.

Le va et vient continuel entre le plan général et le plan particulier est une des données inhérentes au projet de Humboldt. On le retrouve dans les principes méthodologiques adoptés pour la description des structures des langues :

- La comparaison qui permet d'éclairer le général à partir du particulier et de découvrir le particulier depuis le général.

- La méthode inductive qui revient à rechercher les lois internes de chaque langue et favorise sa caractérisation.

D'avoir pensé le langage dans sa dimension anthropologique a eu pour conséquence de toujours viser l'organisation de l'étude des particularités linguistiques dans le cadre d'une étude générale qui doit se comprendre comme « une réflexion unifiante sur la diversité des langues ${ }^{37} »$.

\section{Conclusion}

80 La linguistique cognitive et ses rapports avec Wilhelm von Humboldt sont un sujet qui est loin d'être épuisé. Devant l'ampleur de la tâche, j'ai cherché à ne traiter que ce qui est attesté par les textes des auteurs concernés à la fois pour les filiations et le débat sur l'approche de la diversité des langues dans la linguistique cognitive. Nous avons constaté que Humboldt est une des sources de la linguistique cognitive (à des degrés divers), en Europe et aux Etats-Unis. Sa pensée est réduite à quelques concepts parfois malmenés, sans référence à la globalité de sa théorie du langage. Cette histoire des idées est encore à compléter. Il conviendrait de mener plus loin l'analyse contrastive des espaces culturels, 
scientifiques et linguistiques. Elle permettrait d'appréhender de façon plus complète et dynamique cette linguistique cognitive qui se développe aux quatre coins de la planète, dans de nombreuses langues et qui a de toute évidence des liens, même ténus avec la théorisation humboldtienne.

Enfin cette filiation entre Humboldt et la linguistique cognitive est peut-être tout simplement à consommer. En effet, la théorisation de la diversité des langues mise en œuvre à un plan de recherche comparative qui combine une linguistique de la langue à une linguistique du discours n'a pas été analysée dans sa globalité, ni traitée. C'est peutêtre là toute l'actualité des textes humboldtiens. Ces œuvres sont à lire pour alimenter aujourd'hui la réflexion sur les rapports entre la langue et la culture sans rien exclure des liens à théoriser entre une étude de la structure des langues et une étude des discours.

\section{BIBLIOGRAPHIE}

CEuvres \& traductions de Humboldt

Gesammelte Schriften, A. Leitzmann, 17 vol., Berlin, B. Behr's Verlag.

Ueber den Dualis, in Gesammelte Schriften VI, Über die Sprache, Jürgen Trabant (Hrsg.), Tübingen und Basel, Francke Verlag, 1994.

Brinton Daniel, « Translation of Wilhelm von Humboldt's on the verb in American Languages ", Proceedings of American Philosophical Society 22, 4, 1885.

Caussat Pierre, Introduction à l'oeuvre sur le Kavi et autres essais, Paris, Seuil, 1974.

Harden T., Farelly D. (eds), Wilhelm von Humboldt Essays on language, traduction de John Wieczorek \& Ian Roe, Francfort/Main, Peter Lang, 1997.

Thouard Denis, Sur le caractère national des langues, Essais, Seuil, 2000.

Tonnelé Alfred, De l'origine des formes grammaticales et de leur influence sur le développement des idées suivi de Lettre à M. Abel Rémusat, Editions Ducros, 1969, Première édition 1859.

Autres références

Auroux Sylvain, « Linguistique et anthropologie en France », in S. Auroux (dir.), Histoire de l'anthropologie : XVI - XIX $X^{e}$ siècles, Paris, Klincksieck, 1984, 291-318.

Brown Penelope, « Anthropologie cognitive », Anthropologie et sociétés, Vol. 23, n³,

«L'ethnolinguistique », Québec, Université Laval, 2000. En ligne sur le site de la revue :

www.erudit.org/revue/as/1999/v23/n3/index.html

Brown Roger Langham, Wilhelm von Humboldt's conception of linguistic relativity, La Hague, Paris, Mouton, 1967.

Caussat Pierre, « La confrontation entre Analyse et Synthèse : Humboldt avocat de la révolution kantienne face à la pensée héritée de Condillac », in Kodikas/Code, "Sprachdenken zwischen Berlin und Paris, Wilhelm von Humboldt. La pensée linguistique entre Berlin et Paris, Wilhelm von Humboldt », Vol. 27, n¹/2, Tübingen, Günter Narr Verlag, 2004, 51-64. 
Chabrolle-Cerretini Anne-Marie (dir.), Dossier d'HEL, « Wilhelm von Humboldt. Editer et lire Humboldt », supplément électronique à la revue HEL, Paris, SHESL, 2002, disponible sur Internet : http://htl.linguist.jussieu.fr/num1/num1.htm

Chabrolle-Cerretini Anne-Marie, « Les références à Humboldt dans la linguistique française contemporaine ", Kodikas/Code, «Sprachdenken zwischen Berlin und Paris, Wilhelm von Humboldt. La pensée linguistique entre Berlin et Paris, Wilhelm von Humboldt », Vol. 27, $n^{\circ} 1 / 2$, Tübingen, Günter Narr Verlag, 2004. 103-117.

Chabrolle-Cerretini Anne-Marie (éd.), « Wilhelm von Humboldt, les langues et sa théorie du langage », Verbum, tome XXVII, n¹-2, 2005.

Chabrolle-Cerretini Anne-Marie, Histoire d'un concept linguistique : la « vision du monde » de Wilhelm von Humboldt, ENS Lyon, Coll. Langages, à paraitre novembre 2007.

Chomsky Noam, Le langage et la pensée, (1968), trad. française, Paris, Payot, 1969.

Chomsky Noam, Nouveaux horizons dans l'étude du langage et de l'esprit, (2000), trad. française, Paris, Stock, 2005.

Darnell Regina, « Benjamin Lee Whorf et les fondements boasiens de l'ethnolinguistique contemporaine ", Anthropologie et sociétés, « L'ethnolinguistique », Vol. 23, n³, Québec, Université Laval, 2000. En ligne $:$ http://www.erudit.org/revue/as/1999/v23/n3/015617ar.pdf

Di Cesare Donatella, Humboldt, La diversità delle lingue, Roma-Bari, Editori Laterza, Biblioteca Universale Laterza, 1993.

Dupuy Jean-Pierre, Aux origines des sciences cognitives, Paris, La Découverte/Poche, 1999.

Fuchs Catherine (dir.), La linguistique cognitive, Ophrys, 2004.

Fuchs Catherine, Robert Stéphane (éds), Diversité des langues et représentations cognitives, Paris, Ophrys, 1997.

Gipper Helmut, « Le principe de relativité linguistique et les indiens hopis », in Gardin Bernard \& Marcellesi Jean-Baptiste (éds), Sociolinguistique : approches, théories, pratiques, vol.1, Paris, Rouen, Presses de l'Université, \& Paris, PUF, 1980, 215-224.

Hymes Dell, « Models of interaction of Language and Social Live », Directions in sociolinguistics. The ethnography of communication, New York, Holt, Rinehart \& Winston, 1972, 35-71.

Hymes Dell, Vers la compétence de communication, Paris, Hatier/Didier, 1991.

Joseph John E., From Whitney to Chomsky, Essays in the history of American linguistics, Amsterdam/ Philadelphia, John Benjamins Publishing Company, 2002.

Kokochkina Elena, « De Humboldt à Potebnja : évolution de la notion d"“Innere Sprachform” dans la linguistique russe », Cahiers de Ferdinand de Saussure, n53, Genève, Droz, 2000, 101-122.

Lee Penny, « Whorf's Linguistic relativity », in Martin Pütz \& Marjolijn H. Verspoor (eds), Explorations in linguistic relativity, Amsterdam/Philadelphia, J. Benjamins Publishing Company, $2000,45-68$.

Lenoir Frédéric, Tardan-Masquelier Ysé, Le livre des sagesses, l'aventure spirituelle de l'humanité, Paris, Bayard, 2002.

Lucy John A., Language, diversity and thought, Cambridge University Press, 1992.

Meschonnic Henri, « Théorie du langage, théorie politique, une seule stratégie (Humboldt, Saussure selon Chomsky) », in Pour la poétique V, Poésie sans réponse, Paris, Gallimard, 1978. 
Niemeier Susanne,Dirven René, Mercator Gerhard \& Lucy John A. (eds) Evidence for Linguistic Relativity, Series IV-Current issues in linguistic theory, Amsterdam/Philadelphia, John Benjamins Publishing Company, 2000.

Normand Claudine, «La question d'une science générale »,in S. Auroux (dir.), Histoire des idées linguistiques, Tome 3, Liège, Mardaga, 2000, 442.

Pütz Martin \& Verspoor Marjolijn H. (eds), Explorations in Linguistic Relativity, Series IV-Current issues in linguistic theory, Amsterdam/Philadelphia, John Benjamins Publishing Company, 2000.

Sapir Edward, Linguistique, Paris, Gallimard, Folio Essais, 1991.

Sériot Patrick, « Oxymore ou malentendu ? Le relativisme universaliste de la métalangue sémantique naturelle universelle d'Anna Wierszbicka », Cahiers de Ferdinand de Saussure, $\mathrm{n}^{\circ}$ 57, Genève, 2005, 23-43.

Tetel Andresen Julie, Linguistics in America 1769-1924. A critical history, London and New-York, Routledge, 1990.

Trabant Jürgen, Humboldt ou le sens du langage, Liège, Mardaga, 1995.

Trabant Jürgen, Traditions de Humboldt, Paris, Éditions de la Maison des Sciences de l'homme, 1999.

Trabant Jürgen, « Le courant humboldtien », in S. Auroux(dir.), Histoire des idées linguistiques, Tome 3, Sprimont, Mardaga, 2000.

Trabant Jürgen, « L'édition des œuvres linguistiques de Humboldt. Le sort d'un legs intempestif », Dossier HEL 1, «Éditer et lire Humboldt », [supplément électronique à la revue HEL], Paris, SHESL, 2002. En ligne : http://htl.linguist.jussieu.fr/num1/trabant.htm

Whorf Benjamin Lee, Language, thought, and reality, J. B. Carroll (ed.), Cambridge MA, The M.I.T. Press, 1956.

Whorf Benjamin Lee, Linguistique et anthropologie, traduction française, Paris, Éditions DenoëlGonthier, 1969.

\section{NOTES}

1. Tetel Andresen Julie, 1990.

2. Di Cesare Donatella, 1993, 1999.

3. Auroux Sylvain, 1984.

4. Trabant Jürgen, 2000, p. 318.

5. Brinton Daniel, "Translation of Wilhelm von Humboldt's on the verb in American Languages », Proceedings of American Philosophical Society 22, 4, 1885.

6. Kokochkina Elena, 2000, p. 101.

7. Voir Trabant Jürgen, 2002.

8. Dupuy Jean-Pierre, 1999.

9. Fuchs Catherine, 2004, p. 3.

10. Cf. dans ce numéro G. Achard-Bayle.

11. Cf. dans ce numéro A. Koselak.

12. Kokochkina Elena, 2000.

13. Cf. dans ce numéro Arkadiusz Koselak.

14. Cf. entre autres textes Sériot Patrick, 2005.

15. Meschonnic Henri, 1978. 
16. En fait il est admis que la linguistique de Saussure correspond à la partie descriptive des structures des langues chez Humboldt.

17. Meschonnic Henri, 1978, pp. 332-333. Cf. Ueber die Verschiedenheiten der menschlichen Sprachbaues", Gesammelte Schriften, Band VII, p.99. trad. française "C'est pourquoi elle est appelée à faire, à partir de moyens finis, un usage infini, puisque aussi bien c'est une seule et même force qui produit à la fois la pensée et la langue.», Introduction à l'œuvre sur le Kavi, trad. Pierre Caussat, Paris, Editions du Seuil, p. 246.

18. Chomsky Noam, 1969, p. 33.

19. Chomsky Noam, 2005, p. 44.

20. Fuchs Catherine, Robert Stéphane, 1997.

21. Joseph John E., p. 71.

22. Chabrolle-Cerretini Anne-Marie, à paraître novembre 2007.

23. Caussat Pierre, 2004, p. 58.

24. Sapir Edward, 1991, p. 34.

25. Sapir Edward, 1991, p. 37.

26. Sapir Edward, 1991,p. 56.

27. Whorf Benjamin Lee, Traduction française, 1969, p. 193.Whorf Benjamin Lee, «Language, mind and reality ", Language, thought, and reality, Edited by J. B. Carroll, The M.I.T. Press, 1956, p. 252.Les références du texte en anglais sont données à la suite, la traduction française ayant été beaucoup discutée.

28. Whorf Benjamin Lee, Traduction française, Paris, 1969, p. 143. Whorf Benjamin Lee, "Linguistices as an exact science », Language, thought, and reality, Edited by J. B. Carroll, The M.I.T. Press, 1956, p. 221.

29. Whorf Benjamin Lee, Traduction française, Paris, 1969, p. 96. Whorf Benjamin Lee, « The relation of habitual thought and behavior to language ", Language, thought, and reality, Edited by J. B. Carroll, The M.I.T. Press, 1956, p. 147.

30. Whorf Benjamin Lee, Traduction française, Paris, 1969, p. 73. Whorf Benjamin Lee, «The relation of habitual thought and behavior to language», Language, thought, and reality, Edited by J. B. Carroll, The M.I.T. Press, 1956, p. 135.

31. Whorf Benjamin Lee, Traduction française, Paris, 1969, pp. 76-77. Whorf Benjamin Lee, «The relation of habitual thought and behavior to language», Language, thought, and reality, Edited by J. B. Carroll, The M.I.T. Press, 1956, pp. 136-137.

32. «Isolat d'expérience » mot traduit de P. Lee « isolate of experience », Lee Penny, "Whorf's Linguistic relativity », Explorations in linguistic relativity, volume 199, Edited by Martin Pütz, Marjolijn H. Verspoor, J. Benjamins Publishing Company, Amsterdam/Philadelphia, 2000. On lit chez B. L. Whorf "we escribe a semifictitious isolation to parts of experience ", "Languages and logic » Language, thought, and reality, Edited by J. B. Carroll, The M.I.T. Press, 1956, p. 240.

33. Whorf Benjamin Lee, Traduction française, Paris, 1969, p. 196. Whorf Benjamin Lee, "Language, mind and reality", Language, thought, and reality, Edited by J. B. Carroll, The M.I.T. Press, 1956, p. 253.

34. Texte non traduit en français. Whorf Benjamin Lee, «Language : plan and conception of arrangement ", Language, thought, and reality, Edited by J. B. Carroll, The M.I.T. Press, 1956, pp. 126-133.

35. Gipper Helmut, 1980.

36. La théosophie est une forme spécifique d'ésotérisme dont le précurseur fut Paracelse (1493-1541). Au XIX ${ }^{\mathrm{e}}$ siècle de nouveaux courants s'imposent comme l'occultisme qui atteignit son apogée avec la création par H.P. Blavatsky de la création en 1875 de la Société théosophique dont les objectifs étaient de : «former le noyau de la fraternité universelle; d'étudier dans leurs convergences les religions, la philosophie et la science et d'explorer les lois encore inexpliquées de la nature et les pouvoirs cachés de l'homme » ((Lenoir Frédéric, Tardan-Masquelier, 2002, p. 
1735), (Cf. p.1567, pp. 1730-1742)). Selon J. Joseph, les archives de Whorf à Yale montrent que le linguiste baignait dans la théosophie depuis son plus jeune âge par son père et que ses travaux sur les langues amérindiennes portent l'empreinte de cette approche du monde.

37. Normand Claudine, 2000, p. 442.

\section{RÉSUMÉS}

Cet article étudie la ou les filiations, ou encore la postérité de Humboldt, même s'il s'agit dans les faits d'une filiation complexe ou d'une postérité plus « embrouillée » qu'il n'y paraît; elle montre notamment quelle influence a exercée Humboldt sur la linguistique nord-américaine, notamment au travers de ses deux grands représentants anthropolinguistes: Sapir et Whorf; ceci posé, elle analyse, à la fois en élargissant le propos et en revenant à ce qui caractérise le mieux le modèle humboldtien, dans quel sens, là encore plus complexe qu'il n'y parait, il faut entendre le lien (non « représentationnaliste ») qui unit langue et culture(s) - le pluriel est plus que de rigueur dans le plaidoyer de l'auteur et de son illustre modèle en faveur de la diversité.

This article goes into the Humboldt's affiliations or posterity, even though we should consider that we have here complex affiliations and a posterity more " confused " than it seems. In this spirit, the article shows Humboldt's influence on North American linguistics, and in particular on the two great representatives of anthropological linguistics: Sapir and Whorf. The article next aims at (1) enlarging the exposé and (2) returning to what best characterizes the Humboldtian model, in order to analyse how we should understand the link uniting language and culture(s). Here again, however, it is more complex than it appears, knowing on the one hand that this link between language and culture(s) is not « representationalist ", and that cultures must indeed be envisaged in the plural, in all their diversity.

\section{INDEX}

Mots-clés : langue/culture, Humboldt, Sapir, Whorf, Chomsky, vision du monde, anthropologie, filiation

Keywords : language/culture, World of View, anthropology

\section{AUTEUR}

\section{ANNE-MARIE CHABROLLE-CERRETINI}

CELTED, Université Paul Verlaine- Metz-CELTED, \& UMR CNRS 7597, PARIS 7 